\title{
Induction of interleukin-10 by HIV antigens in peripheral mononuclear cells of health care workers after occupational exposure to HIV-1-positive blood
}

D.S.S. Rodrigues, M.K.C. Brunialti, E.A.S. Medeiros,

R.S. Diaz, G. Turcato and R. Salomao

\author{
Disciplina de Doenças Infecciosas e Parasitárias, \\ Escola Paulista de Medicina, Universidade Federal de São Paulo, \\ São Paulo, SP, Brasil
}

\section{Correspondence \\ R. Salomao \\ Disciplina de Doenças Infecciosas e Parasitárias, EPM, UNIFESP \\ Rua Pedro de Toledo, 781 04039-032 São Paulo, SP \\ Brasil \\ E-mail: rsalomao- \\ dipa@ pesquisa.epm.br}

Research supported by PRO NEX (No. 41.96.0943.00) from the Ministry of Science and Technology. D.S.S. Rodrigues is the recipient of a fellowship from CAPES. Publication supported by FAPESP.

Received June 21, 2000 Accepted April 1, 2002

\section{Abstract}

Evaluation of HIV-induced IL-2 production by peripheral blood mononuclear cells (PBMC) and HIV-specific T helper and cytotoxic T lymphocyte (CTL) responses in health care workers (HCW) occupationally exposed to HIV reveals a high rate of response to HIV among non-seroconverters. IL-10 is also known to interfere with HIV infection in vitro. To evaluate the induction of IL-10 by HIV antigens in $\mathrm{HCW}$ occupationally exposed to HIV, $18 \mathrm{HCW}$ with percutaneous injury were enrolled in this study, 9 of them exposed to HIV-contaminated blood, and 9 exposed to HIV-negative blood. PBMC were incubated on plates coated with HIV-1 antigens, and IL-10 was measured in supernatants by ELISA. Five of nine HCW exposed to HIV-contaminated blood presented HIV-induced IL-10. Two of nine HCW exposed to HIV-negative source patients also had detectable levels of HIV-induced IL-10, one of them in the sample obtained on the day of accidental exposure. There was a relationship between the type of device involved in injury and IL-10 production. Individuals exposed to hollow needles or scalpels presented HIV-induced IL-10, whereas those exposed to solid needles and to digital puncture did not, suggesting a relationship between infectious load and IL-10. Although occupational exposure to HIV leads to a low rate of seroconversion, these individuals can develop an antigen-specific immune response characterized in our study by induction of IL-10 in PBMC in vitro.
The rate of HIV infection of health care workers $(\mathrm{HCW})$ associated with occupational exposure to contaminated blood or body fluids is estimated to be $0.3 \%$ (1). Risk factors associated with higher rates of HIV-1 transmission are deep injury, visible blood in the

\section{Key words}

- HIV-1

- Interleukin-10

- Needlestick injury

- Occupational exposure

- Health care worker device involved in the accident, procedures involving needles in arteries or veins, and terminal illness of the source patient (2).

Despite the low rate of seroconversion, $\mathrm{HCW}$ frequently develop an HIV-specific response following occupational exposure. 
HIV-specific production of interleukin (IL)2 and cytotoxic T lymphocyte (CTL) activity has been demonstrated in HCW accidentally exposed to HIV, suggesting that the contact with the virus may be more frequent than expected, and the immune response is possibly a major determinant of protection against HIV infection in this setting (3-5).

IL-10 is a cytokine derived from monocytes, $\mathrm{B}$ and $\mathrm{T}$ lymphocytes that is a potent macrophage deactivator (6). The role of this cytokine in HIV infection has been the subject of many studies. Although it has been shown that as the disease progresses IL-10 may be involved in the down-regulation of the immune response (7), there is also evidence that IL-10 may protect macrophages from HIV-1 infection in vitro (8). Interestingly, the enhancement of HIV-1 replication in macrophages has also been attributed to IL-10 (9).

The aims of the present study were to evaluate the production of IL-10 by peripheral blood mononuclear cells (PBMC) from HCW occupationally exposed to HIV-1 in response to HIV antigens and to evaluate the relationship between the presence of HIVinduced IL-10 production and the characteristics of each accident.

Eighteen HCW were enrolled in the study between July 1997 and February 1998. The research was approved by the University Ethics Committee and written informed consent was obtained from all participants. The volunteers were recruited from Hospital São Paulo, the University Hospital of Universidade Federal de São Paulo (UNIFESP). Nine HCW had been percutaneously exposed to blood from HIV-1-negative source patients and nine to blood from HIV-positive patients.

Demographic HCW data included age, sex, occupation, and other possible exposures to HIV-1 besides occupational exposure. Source patient data included presence or absence of symptoms, peripheral blood CD4 $\mathrm{T}$ cell counts and plasma viral load and clinical outcome over two months of followup after the episode. The accident was characterized by the type of device involved (scalpel, hollow or solid needle), presence of blood, and use of gloves by the HCW. The use of prophylactic AZT was also recorded. HCW were followed up for 6 months. Plasma viral loads and antibody testing for HIV-1 (ELISA) were performed at days 0, 30, 90, and 180 after exposure.

For the induction of IL-10, in vitro blood samples were collected over a period of six months after exposure, and PBMC were obtained by gradient centrifugation over Ficoll (Pharmacia-Biotech, Uppsala, Sweden). PBMC were resuspended at $2 \times 10^{6}$ cells $/ \mathrm{ml}$ in RPMI 1640 medium (Sigma, St. Louis, MO, USA) supplemented with $10 \%$ autologous serum. Medium was used as negative control, whereas the phytohemagglutinin (PHA) stimulus was considered as positive control. For HIV-specific stimulation we used an ELISA plate coated with HIV-1 antigens (Hemobio HIV-1, Embrabio, São Paulo, SP, Brazil). This plate was successfully used for the induction of HIV-1 antibody production in vitro (10), and in our experience has permitted us to discriminate HIV-positive patients from seronegative healthy volunteers. We tested 23 HIV-positive asymptomatic patients taking no antiretroviral treatment, and $21 \mathrm{HIV}$-seronegative healthy volunteers and found HIV-induced IL-10 to be higher in HIV-positive patients (median $34.6 \mathrm{pg} / \mathrm{ml}$, range $0-242 \mathrm{pg} / \mathrm{ml}$ ) than in healthy volunteers (median $0 \mathrm{pg} / \mathrm{ml}$, range $0-61 \mathrm{pg} / \mathrm{ml}$ ) ( $\mathrm{P}=0.0008$, Mann-Whitney test). Supernatants were collected after $48 \mathrm{~h}$ of incubation at $37^{\circ} \mathrm{C}$ in a $5 \% \mathrm{CO}_{2}$ atmosphere. Induction of IL-10 was considered to be HIV-specific when the levels obtained by HIV stimulation were at least two times that of the negative control.

IL-10 was measured in the supernatants by ELISA. Monoclonal antibodies and recombinant IL-10 were obtained from PharMingen (San Jose, CA, USA). Samples from 
the same HCW were tested simultaneously. The sensitivity of the test was $5 \mathrm{pg} / \mathrm{ml}$.

Plasma load of HIV RNA was quantitated using the Nucleic Acid-Based Amplification System (Organon Teknika, Boxtel, The Netherlands) according to manufacturer instructions. The lowest detection limit was 400 copies $/ \mathrm{ml}$.

No HIV seroconversion or positive RNA viral load was detected among the HCW enrolled in the study.

Among the six HIV-positive source pa- tients, five had AIDS and two died 2 months after exposure. One HIV-positive patient was the source patient of four HCW enrolled in this study. There was visible blood in all accidents. The HCW were always using latex gloves and in two instances were using double gloves when the injury occurred.

Tables 1 and 2 show the levels of IL-10 after HIV stimulation, as well as values for negative (medium) and positive control (PHA) in HCW exposed to HIV-1-positive and HIV-1-negative source patients. PHA-

Table 1. Detection of IL-10 in supernatants of PBMC from health care workers (HCW) exposed to an HIVpositive source patient at the time of exposure and during follow-up.

\begin{tabular}{|c|c|c|c|c|c|c|c|c|c|c|c|c|}
\hline \multirow[t]{2}{*}{$\mathrm{HCW}$} & \multicolumn{3}{|c|}{ TO } & \multicolumn{3}{|c|}{$\mathrm{T} 1$} & \multicolumn{3}{|c|}{ T3 } & \multicolumn{3}{|c|}{ T6 } \\
\hline & Ctl & HIV & PHA & Ctl & HIV & PHA & $\mathrm{Ctl}$ & HIV & PHA & Ctl & HIV & PHA \\
\hline 1 & - & - & - & nd & nd & 96.6 & 23.6 & 21.2 & 172.9 & nd & 22.8 & 324.5 \\
\hline 2 & - & - & - & - & - & - & 45.4 & 146.8 & 750.9 & - & - & - \\
\hline 3 & - & - & - & - & - & & nd & nd & 263.0 & 121.1 & 97.0 & 911.9 \\
\hline 4 & - & - & - & nd & nd & 114.0 & - & - & - & nd & nd & 146.9 \\
\hline 5 & - & - & - & - & - & - & 21.6 & 32.9 & 269.6 & nd & 120.7 & 301.8 \\
\hline 6 & 7.2 & 4.2 & 1589.2 & 26.7 & 115.4 & 386.6 & nd & nd & 125.8 & 10.2 & 21.4 & 527.1 \\
\hline 7 & 109.6 & 113.4 & 945.6 & 102.1 & 105.0 & 794.9 & - & - & - & 13.4 & 12.5 & 535.8 \\
\hline 8 & - & - & - & nd & 3.7 & 660.0 & - & - & - & 105.9 & 107.6 & 236.0 \\
\hline 9 & - & - & - & - & - & - & nd & 56.1 & 899.3 & 10.5 & 94.6 & 830.2 \\
\hline
\end{tabular}

Data are reported as $\mathrm{pg} / \mathrm{ml}$. Values in bold indicate a positive response to HIV-1 antigens (2-fold increase over control). - = not measured; nd = not detectable; $\mathrm{CtI}=$ negative control (medium); PHA = positive control (phytohemagglutinin stimulus); T0, T1, T3 and T6: baseline and 1, 3 and 6 months after exposure.

Table 2. Detection of IL-10 in supernatants of PBMC from health care workers (HCW) exposed to an HIVnegative source patient at the time of exposure and during follow-up.

\begin{tabular}{|c|c|c|c|c|c|c|c|c|c|c|c|c|}
\hline \multirow[t]{2}{*}{$\mathrm{HCW}$} & \multicolumn{3}{|c|}{ TO } & \multicolumn{3}{|c|}{$\mathrm{T} 1$} & \multicolumn{3}{|c|}{ T3 } & \multicolumn{3}{|c|}{ T6 } \\
\hline & CtI & HIV & PHA & Ctl & HIV & PHA & CtI & HIV & PHA & CtI & HIV & PHA \\
\hline 1 & - & - & - & 199.1 & 147.2 & 711.0 & 12.8 & 14.2 & 178.8 & - & - & - \\
\hline 2 & - & - & - & 11.8 & 12.3 & 330.8 & 16.9 & 13.9 & 223.7 & nd & nd & 23.6 \\
\hline 3 & - & - & - & nd & nd & 1117.0 & 3.7 & 21.6 & 662.9 & - & - & - \\
\hline 4 & nd & nd & 832.1 & 52.6 & 54.3 & 1257.8 & - & - & - & nd & nd & 950.4 \\
\hline 5 & 17.2 & 88.0 & 147.8 & 146.5 & 167.8 & 902.5 & - & - & - & nd & nd & 356.5 \\
\hline 6 & 139.8 & 160.3 & 1861.7 & 120.5 & 135.2 & 2178.0 & 121.4 & 113.0 & 2157.1 & 102.5 & 103.1 & 1132.1 \\
\hline 7 & 233.5 & 284.9 & 4334.1 & - & - & - & 117.2 & 113.4 & 2671.6 & 105.9 & 107.6 & 236.0 \\
\hline 8 & 52.4 & 85.4 & 1224.7 & - & - & - & 44.2 & 66.3 & 338.4 & - & - & - \\
\hline 9 & - & - & - & - & - & - & nd & nd & 384.1 & - & - & - \\
\hline
\end{tabular}

Data are reported as $\mathrm{pg} / \mathrm{ml}$. Values in bold indicate a positive response to HIV-1 antigens (2-fold increase over control). - = not measured; $\mathrm{nd}=$ not detectable; $\mathrm{CtI}=$ negative control (medium); PHA = positive control (phytohemagglutinin stimulus); T0, T1, T3 and T6: baseline and 1, 3 and 6 months after exposure. 
induced IL-10 did not differ between HCW groups. Five of nine HCW exposed to an HIV-1-positive source patient had detectable HIV-induced IL-10 (100\% increase over negative control). In one exposed individual, positive results were obtained in samples collected 1 and 6 months after exposure; in two the positive sample was collected at 6 months. The fourth exposed HCW had positive results at months 3 and 6 , and the last one at month 3 (Table 1). Two of nine HCW exposed to an HIV-negative source patient also had detectable levels of HIV-induced IL-10, one in the sample collected on the day of occupational exposure and the other in the sample collected at 3 months (Table 2).

Among the accidents involving HIV-positive source patients in this study, four HCW were exposed to the same asymptomatic patient (HCW 1, 2, 3 and 4; Table 1) and five to other AIDS patients. Induction of IL-10 was observed in two of the former and in three of the latter. CD4 and viral load were obtained from only a small proportion of HIV-1-positive source patients, which precludes any analysis of the correlation of these parameters and HIV-induced IL-10 in recipient $\mathrm{HCW}$. Five HCW did not receive antiretroviral post-exposure prophylaxis and four received AZT. IL-10 was found in three HCW of the first group and in two of the second one. The type of device involved in the injury differed between HCW exposed to an HIV-positive source that had HIV-induced IL-10 production and those without HIVinduced IL-10 production. The devices involved in the accidents without IL-10 production were solid needles (HCW 3 and 4) or needles used for digital punctures for determination of glucose levels (HCW 7 and 8). The devices involved in the accidents of the five HCW with HIV-induced IL-10 production were scalpels (HCW 2) or hollow-bore needles used for phlebotomy (HCW 1, 5, 6, 9) (Table 1).

The results of this study show that production of HIV-1-induced IL-10 was found in five of nine HCW exposed to HIV-1contaminated blood and in two of nine $\mathrm{HCW}$ exposed to HIV-negative blood. In the first group IL-10 induction was mainly observed between 3 and 6 months after exposure. One HCW of the second group had a positive result in the sample obtained on the day of exposure. Thus, this result may be considered as a) false positive, b) not related to the accident, or c) perhaps related to another previous exposure.

These findings agree with studies that reported HIV-induced IL-2 production in $75 \%$ of HCW occupationally exposed to HIV compared to $24 \%$ in the control group (4). CTL activity has also been shown in $35 \%$ of exposed subjects and in no subject of the control group (5).

In a case-control study, Cardo et al. (2) showed that increased risk for HIV infection after blood exposure is related to a larger volume of blood and, possibly, a higher HIV load in the source patient's blood.

In our study, we observed that the medical devices involved in HCW injuries with HIV-positive source patients differed between those with HIV-induced IL-10 and those without HIV-induced IL-10. In the first group percutaneous exposure involved either hollow-bore needles used for phlebotomy or a scalpel. In the latter group occupational exposure was by solid needle or by hollow-bore needles used for digital puncture. Thus, if we assume that the former group was exposed to a larger amount of blood, a correlation between large blood volumes and IL-10 may be considered. This hypothesis is supported by the findings of the cluster of the four HCW exposed to the same HIV-1-infected source patient. Interestingly, in this cluster, HIV-induced IL-10 was seen in two HCW with either lancet injuries (HCW 2) or solid plus hollow-bore needle injuries (HCW 1), but was not seen in the other two HCW (HCW 3 and 4) whose injuries involved solid needles. No other correlation was seen between the character- 
istic of the exposure and IL-10 production. In all cases HCW were using gloves and bleeding was present.

The role of IL-10 in HIV-1 infection remains controversial. It is likely that, by down-regulating proinflammatory cytokines, IL-10 inhibits HIV-1 replication. It was observed that IL-10 is produced during HIV-1 infection and that IL-10 inhibits viral replication in macrophages in vitro $(8,11,12)$. On the other hand, the enhancement of virus replication is also attributed to IL-10 (9). It has been suggested that, in amounts not large enough to inhibit TNF- $\alpha$, IL-10 may increase virus replication. Moreover, it has also been shown that IL-10 may be impor- tant in cellular immune dysfunction in HIVinfected patients (13).

The demonstration of HIV-induced IL-10 in HCW occupationally exposed to HIV-1 in this study supports the idea that specific immune responses are seen at much higher rates than seroconversion. The role of IL-10 in this setting remains to be established.

\section{Acknowledgments}

We are indebted to Dr. Esper Georges Kallás for a critical review of the manuscript.

\section{References}

1. Centers for Disease Control (1995). Casecontrol study of HIV seroconversion in health-care workers after percutaneous exposure to HIV-infected blood - France, United Kingdom, and United States, J anuary 1988-August 1994. Morbidity and Mortality Weekly Report, 44: 929-933.

2. Cardo DM, Culver DH, Ciesielski CA, Srivastava PU, Marcus R, Abiteboul D, Heptonstall J , Ippolito G, Lot F, McKibben PS \& Bell DM (1997). A case-control study of HIV seroconversion in health care workers after percutaneous exposure. New England J ournal of Medicine, 337: 14851490.

3. Pinto LA, Landay AL, Berzofsky JA, Kessler HA \& Shearer GM (1997). Immune response to human immunodeficiency virus (HIV) in health care workers occupationally exposed to HIV-contaminated blood. American J oumal of Medicine, 102: 21-24.

4. Clerici M, Levin J M, Kessler HA, Harris A, Berzofsky J A, Landay AL \& Shearer GM (1994). HIV-specific T-helper activity in seronegative health care workers exposed to contaminated blood. J oumal of the American Medical Association, 271: 42-46.
5. Pinto LA, Sullivan J , Berzofsky J A, Clerici M, Kessler HA, Landay AL \& Shearer GM (1995). Env-specific cytotoxic T lymphocyte responses in HIV seronegative health care workers occupationally exposed to HIV-contaminated body fluids. J oumal of Clinical Investigation, 96: 867-876.

6. Mosmann TR (1994). Properties and functions of interleukin-10. Advances in Immunology, 56: 1-27.

7. Clerici M \& Shearer GM (1993). A $\mathrm{TH} 1 \rightarrow \mathrm{TH} 2$ switch is a critical step in the etiology of HIV infection. Immunology Today, 14: 107-111.

8. Akridge RE, Oyafuso LKM \& Reed SG (1994). IL-10 is induced during HIV-1 infection and is capable of decreasing viral replication in human macrophages. J ournal of Immunology, 153: 5782-5789.

9. Weissman D, Poli G \& Fauci AS (1995). IL10 synergies with multiple cytokines in enhancing HIV production in cells of monocytic lineage. J ournal of Acquired Immune Deficiency Syndromes, 9: 442449.

10. Caterino-de-Araujo A, De Los SantosFortuna E \& Grumach AS (1991). An alternative method for in vitro production of
HIV-1-specific antibodies. Brazilian J ournal of Medical and Biological Research, 24: 797-799.

11. Kollmann TB, Pettoello-Mantovani M Katopodis NF, Hachamovitch M, Rubinstein A, Kim A \& Goldstein H (1996). Inhibition of acute in vivo human immunodeficiency virus infection by human interleukin 10 treatment of SCID mice implanted with human fetal thymus and liver. Proceedings of the National Academy of Sciences, USA, 93: 3126-3131.

12. Kootstra NA, Van't Wout A, Huisman HG, Miedema F \& Schuitemaker H (1994). Interference of interleukin-10 with human immunodeficiency virus type 1 replication in primary monocyte-derived macrophages. J ournal of Virology, 68: 69676975.

13. Clerici M, Wynn TA, Berzofsky J A, Blatt $\mathrm{SP}$, Hendrix CW, Sher A, Coffman RL \& Shearer GM (1994). Role of interleukin-10 in $\mathrm{T}$ helper cell dysfunction in asymptomatic individuals infected with the human immunodeficiency virus. J ournal of Clinical Investigation, 93: 768-775. 